\title{
analyse critique de la théorie de consolidation unidimensionnelle de Terzaghi
}

\author{
par \\ F. Tavenas \\ Professeur, Département de Génie civil, \\ Université Laval, Québec \\ M. Brucy \\ Etudiant gradué, Département de Génie civil, \\ Université Laval, Québec \\ J.-P. Magnan \\ IPC, chef de la section des ouvrages en terre, \\ LCPC, Paris \\ P. La Rochelle \\ Professeur, Département de Génie civil, \\ Université Laval, Québec \\ M. Roy \\ Professeur agrégé, Département de Génie civil, \\ Université Laval, Québec
}

\section{Introduction}

Dans l'étude et le dimensionnement des ouvrages construits sur des fondations argileuses, l'évaluation de la vitesse du tassement en fonction du temps a souvent été considérée dans le passé comme un exercice académique d'importance moindre par rapport à l'évaluation de la stabilité et du tassement global de la fondation qui contrôlaient normalement les grandes caractéristiques du projet. Dans ce contexte, la pratique courante des analyses de consolidation est depuis longtemps considérée comme bien établie. Elle fait appel à la théorie de consolidation unidimensionnelle próposée par Terzaghi (1925) pour représenter le phénomène, et aux résultats de l'essai cedométrique pour définir les paramètres caractérisant le comportement de l'argile. Cette méthode, d'un usage très répandu, ne conduit généralement pas à des résultats très satisfaisants mais les conséquences pratiques de ces erreurs de prévisions étaient habituellement limitées.

Avec le développement de la construction de voies de communication et d'installations industrielles on est maintenant amené à édifier des ouvrages de plus en plus importants sur des fondations argileuses de faible qualité. Dans ces conditions on est obligé de faire appel à des techniques de préchargement ou de construction par étapes et la prévision des vitesses de consolidation de la fondation devient un élément déterminant du projet, tant en ce qui concerne l'évaluation de la stabilité durant les diverses phases de construction, que pour la détermination du calendrier de construction et de la viabilité de l'ouvrage. II faut dès lors disposer de méthodes fiables de calcul de la consolidation. La théorie de Terzaghi combinée à l'essai œdométrique, n'ayant pas jusqu'ici conduit à des prévisions toujours satisfaisantes, il est apparu nécessaire d'en réexaminer les fondements théoriques pour voir dans quelle mesure ils correspondent aux conditions réelles du terrain. Par ailleurs, on a pu simuler par analyse numérique les phénomènes réels de consolidation sous forte charge pour montrer dans quelle mesure ils diffèrent des conditions imposées dans l'essai œdométrique classique. De cette façon, la présente étude a pu mettre en évidence les limitations très sérieuses de la méthode classique de calcul de la consolidation.

\section{La théorie de Terzaghi et ses implications}

Ayant constaté que les déformations d'un échantillon d'argile saturé soumis à un accroissement de contrainte $\Delta p$ étaient retardées du fait de la faible perméabilité de l'argile, Terzaghi (1925) a développé une formulation mathématique de ce phénomène. $11 \mathrm{a}$, pour cela, été amené à formuler une série d'hypothèses dont certaines ont des conséquences très importantes sur la possibilité d'appliquer cette théorie à l'étude d'un cas réel. Après avoir rappelé le principe de base du phénomène de consolidation, les différentes hypothèses de Terzaghi seront examinées et leurs conséquences établies.

\subsection{Principe du phénomène de consolidation}

Soit un échantillon de sol, de volume $\mathrm{V}$, qu'on soumet à une variation d'état de contrainte $\Delta \sigma_{1}^{\prime}, \Delta \sigma_{2}^{\prime}, \Delta \sigma_{3}^{\prime}$. Si on admet que ce sol a un comportement élastique isotrope, il en résultera une déformation volumétrique $\Delta \mathrm{V} / \mathrm{V}$ telle que :

$$
\begin{gathered}
\frac{\Delta V}{V}=\varepsilon_{1}+\varepsilon_{2}+\varepsilon_{3}=\frac{1-2 v}{E}\left(\sigma_{i}+\sigma_{2}+\sigma_{3}\right) \\
\text { ou } \frac{\Delta V}{V}=\frac{3(1-2 v)}{E} \Delta \sigma_{\text {oct }}
\end{gathered}
$$

Si on suppose que cet échantillon est parfaitement saturé d'un liquide incompressible et que le chargement est instantané, en état non drainé, on aura alors une déformation volumétrique nulle, ce qui impose $\Delta \sigma_{\text {oct }}^{\prime}=0$ et par conséquent :

$$
\Delta \mathrm{u}=\Delta \sigma_{\mathrm{oct}}
$$

Si on permet maintenant un drainage à la périphérie de l'échantillon où on a donc $\Delta u=0$, le gradient de charge hydraulique entre le centre et la surface de l'échantillon va produire un écoulement de l'eau interstitielle, contrôlé par la perméabilité $k$ du sol. Avec le temps:

- les surpressions interstitielles vont progressivement diminuer de la valeur initiale $\Delta \mathrm{u}=\Delta \sigma_{\text {oct }}$ à la valeur finale 0 ; 
- les contraintes effectives vont augmenter simultanément de la valeur initiale 0 à la valeur finale $\Delta \sigma_{\text {ott }}^{\prime}=\Delta \sigma_{\text {oct }}$ suivant la relation fondamentale $\sigma_{\text {oct }}=\sigma_{\text {oct }}^{\prime}+u$;

- les déformations volumétriques vont donc se développer avec l'accroissement de contraintes effectives suivant la relation (1).

Dans le cas général, tel qu'il se rencontre sur le terrain, toutes les composantes du tenseur de contraintes varient lors du chargement d'une fondation, les déformations volumétriques étant donc gouvernées par l'équation (1). Par ailleurs l'écoulement de l'eau interstitielle est généralement tridimensionnel, si bien que le phénomène de consolidation doit être analysé comme un problème à trois dimensions.

\subsection{Les hypothèses de la théorie de Terzaghi}

Ayant formulé le problème général, Terzaghi (1925) en a développé une solution complète dans un cas particulier simple, soit celui de la consolidation unidimensionnelle d'une couche mince soumise à une charge uniforme $\Delta \sigma_{v}$ de grande étendue. Cette solution, qui est celle utilisée pour l'analyse de tous les problèmes courants de fondations sur dépôts argileux, fait appel à huit hypothèses principales :

1. les déformations de la couche argileuse sont unidimensionnelles :

2. le sol est et demeure saturé ;

3. les grains du sol et le fluide interstitiel sont incompressibles :

4. le sol est homogène:

5. les caractéristiques du sol (module, perméabilité) sont constantes pendant toute la consolidation ;

6. le drainage est unidimensionnel et il obéit à la loi de Darcy:

7. il existe une relation linéaire entre les contraintes effectives et les variations de volume du sol

8. le sol ne présente pas de viscosité structurale, ou consolidation secondaire.

L'équation de conservation de la masse de l'eau dans l'écoulement transitoire qui se développe au cours de la consolidation s'écrit, compte tenu des hypothèses 2 et 3 :

$$
\operatorname{div}\left(\gamma_{w} \vec{v}\right)=\frac{\partial}{\partial t}\left(\gamma_{w} n\right)
$$

ce qui exprime simplement que la quantité d'eau sortie d'un volume est égale à la variation de ce volume.

L'hypothèse 6 d'écoulement unidimensionnel implique que le vecteur vitesse $V$ se réduit à $V_{z} \vec{j}$ ou $\vec{j}$ représente un vecteur unité vertical et l'équation (3) devient:

$$
\frac{\partial V_{z}}{\partial z}=-\frac{\partial n}{\partial t}
$$

Par ailleurs, l'écoulement étant régi par la loi de Darcy qui veut que :

$$
\vec{V}=-k \overrightarrow{g r a d} h
$$

où h représente la charge hydraulique et $\mathrm{k}$ le coefficient de perméabilité du sol, on aura donc en condition unidimensionnelle

$$
v_{z}=-k \frac{\partial h}{\partial z}
$$

avec les notations :

$\mathrm{h}=\frac{\mathrm{u}}{\gamma_{\mathrm{w}}}+\mathrm{z}$ $\mathrm{u}=$ pression interstitielle dans l'élément de sol considéré,

$\gamma_{W}=$ poids volumique de l'eau

$z=$ cote de l'élément de sol considéré.

Compte tenu de $(5)$ et $\left(5^{\prime}\right)$, I'équation (4) peut s'écrire :

$$
-\frac{k}{\gamma_{w}} \frac{\partial^{2} u}{\partial z^{2}}=-\frac{\partial n}{\partial t}
$$

Par ailleurs, Terzaghi suppose implicitement que la variation relative de volume peut s'écrire en fonction de l'indice des vides e du sol sous la forme :

$$
\frac{\partial n}{\partial t}=\frac{\partial}{\partial t}\left(\frac{e}{1+e_{0}}\right) \approx \frac{\partial e}{\partial t}\left(\frac{1}{1+e_{0}}\right)
$$

[Lorsque l'on ne fait pas cette approximation, on obtient une équation légèrement différente (annexe A).]

Pour exprimer la variation d'indice des vides en fonction du temps, Terzaghi fait d'abord appel à l'hypothèse 1 . d'une déformation unidimensionnelle qui implique que les variations de volume du sol sont fonction des seules contraintes effectives verticales et que, à tout instant en cours de consolidation, on a :

$$
\sigma_{v}=\sigma_{v}^{\prime}+u
$$

$\sigma_{\mathrm{v}}$ étant par définition constant, on en déduit

$$
\frac{\partial \sigma_{v}}{\partial t}=\frac{\partial u}{\partial t}
$$

D'autre part, l'hypothèse 7 d'une relation linéaire entre la contrainte effective verticale et l'indice des vides du sol, de la forme

$$
e=e_{o}-a_{v} \Delta \sigma_{v}^{\prime}
$$

permet d'écrire le coefficient de compressibilité $\mathrm{a}_{\mathrm{v}}$ sous la forme :

$$
a_{v}=-\frac{\partial e}{\partial \sigma_{v}^{\prime}}
$$

On a alors, compte tenu de I'hypothèse 8 ,

$$
\frac{\partial e}{\partial t}=\frac{\partial e}{\partial \sigma^{\prime}} \frac{\partial \sigma^{\prime}}{\partial t}
$$

et, en tenant compte de (8) et (9)

$$
\frac{\partial e}{\partial t}=a_{v} \frac{\partial u}{\partial t}
$$

En combinant maintenant (6), (7) et (10) on obtient l'équation différentielle qui régit la consolidation unidimensionnelle :

$$
\frac{\partial u}{\partial t}=\frac{k(1+e) \partial^{2} u}{\gamma_{w} a_{v} \partial z^{2}}
$$

Notant l'analogie avec l'équation de transmission de la chaleur et compte tenu de l'hypothèse 5 , Terzaghi définit le coefficient de consolidation $\mathrm{c}_{\mathrm{v}}$. propriété caractéristique du sol, soit :

$$
c_{v}=\frac{k(l+e)}{\gamma_{w} a_{v}}
$$

Finalement, grâce à l'hypothèse 4 d'homogénéité du sol, Terzaghi arrive à la solution bien connue de l'équation (11), donnant la pression interstitielle $u(z, t)$ en tout point et tout temps dans une couche d'épaisseur $2 \mathrm{H}$ drainée des deux côtés : 
$u(z, t)=\frac{4}{\pi} \Delta \sigma_{v}\left[\sum_{m=0}^{\infty} \frac{1}{2 m+1} \sin \frac{(2 m+1) \pi z}{2 H} \exp \frac{-(2 m+1)^{2} \pi^{2} T v}{4}\right]$

dans laquelle $T_{v}$ est le facteur temps, adimensionnel, de la forme :

$$
T_{v}=\frac{c_{v} t}{H^{2}}
$$

Pour passer de l'équation (13) à la loi de variation du tassement de la couche d'argile en fonction du temps, Terzaghi définit successivement le degré de consolidation local :

$$
u_{z}-1=\frac{u}{u_{i}}
$$

où $u_{i}$ et $u$ désignent respectivement, à la profondeur $z$, les surpressions interstitielles initiale et au temps t, puis, par intégration, le degré de consolidation moyen

$$
U=1-\frac{\int_{0}^{2 H} u d z}{\int_{0}^{2 H} u_{i} d z}
$$

qui peut encore s'écrire, compte tenu de (13), dans le cas où $u_{i}$ est constant dans tout le sol:

$$
U=1-\sum_{m=0}^{\infty} \frac{2}{M^{2}} \exp -M^{2} T
$$

avec :

$M=\frac{\pi}{2}(2 m+1)$

Faisant alors usage de l'hypothèse 7, Terzaghi conclut que l'équation (17) qui donne la variation en fonction du temps de la valeur moyenne de la contrainte effective dans la couche d'argile, donne également la variation du tassement total de cette couche.

\subsection{Limites de validité des hypothèses et leurs conséquences}

On a vu comment chacune des 8 hypothèses énoncées plus tôt intervient pour permettre la formulation de l'équation générale :

$$
\frac{\partial u}{\partial t} \quad=c_{v} \quad \frac{\partial^{2} u}{\partial z^{2}}
$$

et de sa solution (équations 13 et 17).

II convient maintenant d'examiner les limites de validité de ces hypothèses et leurs conséquences sur la possibilité d'arriver à une solution convenable d'un problème réel à partir de l'équation (18).

Lhypothèse 1 de déformation unidimensionnelle a pour avantage évident de permettre d'abord de se libérer de la mesure, toujours délicate, du coefficient de Poisson du sol, et de caractériser la compressibilité du sol par le module mesuré à l'essai œdométrique. Elle permet par ailleurs de simplifier considérablement le calcul des tassements en n'ayant à considérer que la composante verticale du tenseur des contraintes induit dans la fondation.

Elle a cependant une conséquence importante au niveau du calcul de consolidation. En effet, cette hypothèse implique nécessairement que, à l'instant $\mathrm{t}=0$, on $\mathrm{a}$ :

$$
\Delta \mathrm{u}=\Delta \sigma_{\mathrm{v}}
$$

Par conséquent le phénomène consolidation va s'appliquer à une variation de contrainte effective de 0 à $\Delta \sigma_{\mathrm{v}}^{\prime}=\Delta \sigma_{\mathrm{v}}$ et va donc concerner la totalité du tassement.

Au contraire, sous les ouvrages réels l'état de déformation ne sera pas strictement uniaxial. Dans ces conditions, à l'instant $\mathrm{t}=0$, l'équation (2) s'appliquera, $\Delta \sigma_{\text {oct }}$ prenant des valeurs de l'ordre de 0.7 à $0.9 \Delta \sigma_{\mathrm{y}}$. On aura donc instantanément un accroissement de contrainte effective verticale $\Delta \sigma_{v}^{\prime}$ de l'ordre de 10 à 30 pour cent de la charge verticale appliquée et un tassement instantané. C'est pour tenir compte de ce phénomène que Skempton et Bjerrum (1957) ont proposé une correction du tassement œdométrique. Ceci implique également que le phénomène de consolidation ne s'appliquera pas à la totalité du tassement codométrique mais seulement à la partie produite par $\Delta \sigma_{\mathrm{v}}^{\prime}=\Delta \sigma_{\text {oct }}$ soit 70 à 80 pour cent de la charge verticale appliquée.

Les hypothèses 2 et 3 de saturation et dincompressibilité des eléments du sol permettent d'écrire que, à l'instant $\mathrm{t}=\mathrm{O}$, on a $\Delta \mathrm{u}=\Delta \sigma_{\mathrm{v}}$ en condition cedométrique. De manière très générale, il est rare que les sols argileux soient parfaitement saturés d'un liquide incompressible. Au contraire ils contiennent normalement de faibles quantités de gaz, provenant de la décomposition des matières organiques, et qui se présentent quelquefois sous la forme de bulles, mais plus souvent à l'état de dissolution dans l'eau qui devient compressible (Fredlund, 1976).

De ce fait, pour $t=0$, il peut se produire des déformations volumiques qui correspondent nécessairement à une variation des contraintes effectives et donc à des pressions interstitielles initiales inférieures aux valeurs théoriques de l'équation (2). Ce phénomène étudié par Magnan et Dang (1977), conduit donc à une réduction de la portion du tassement total dont le développement est régi par la théorie de consolidation.

Les hypothèses 4 et 5 d'homogénéité et de constance des caractéristiques du sol sont nécessaires pour poser $c_{\mathrm{v}}=$ constante dans l'équation 18 et ainsi permettre une solution exacte de cette équation. Elles sont malheureusement très éloignées de la réalité. En effet il est d'abord très rare de rencontrer une couche d'argile réellement homogène dans la nature. Par ailleurs et surtout, il est maintenant bien établi que les propriétés du sol telles que $k$ et $\mathrm{a}_{\mathrm{v}}$. varient en fonction de l'indice des vides, et donc en cours de consolidation. De ce fait, une couche d'argile qui serait initialement homogène deviendra nécessairement hétérogène en cours de consolidation. Quant à l'hypothèse résultante d'un coefficient de consolidation $c_{y}$ constant, l'examen de la forme de ce coefficient:

$$
c_{v}=\frac{k(1+e) \sigma^{\prime}}{0,434 \gamma_{w} C_{C}}
$$

montre qu'elle a peu de chances d'être exacte puisque $\mathrm{k}$, e, $\sigma^{\prime}$ et $C_{C}$ sont tous variables en cours de consolidation. $L^{\prime}$ erreur introduite en supposant $c_{y}=C^{\text {ste }}$ reste acceptable dans les sols surconsolidés où $\mathrm{C}_{\mathrm{C}}$ est faible et oủ les paramètres sont donc peu variables; elle devient prohibitive lorsque l'argile passe à l'état normalement consolidé en cours de consolidation par suite des variations considérables de $k$, e et $C_{c}$ dans ce cas là.

La solution de Terzaghi ne pourra donc qu'être très approchée dans la plupart des cas. On devra avoir recours à une méthode de calcul permettant de tenir compte de la non-homogénéité et de la variation en cours de consolidation,des propriétés de l'argile pour obtenir une simulation convenable des phénomènes réels. On verra plus loin l'effet des variations de $C_{v}$ sur la qualité de la solution de Terzaghi.

L'hypothèse d'un drainage unidimensionnel obéissant à la loi de Darcy permet d'abord une expression simple des débits, et donc des changements de volume en fonction du temps. On doit évidemment noter que les conditions de géométrie requises pour produire une condition d'écoulement unidimensionel sont rarement réunies dans les fon- 
dations d'ouvrages. On aura généralement un écoulement bidimensionnel, donc plus rapide: Leroueil et al (1978) ont bien mis ce phénomène en évidence. Ici encore, on devra avoir recours à une méthode numérique pour tenir compte de cette diffusion bidimensionnelle et mieux simuler la réalité.

Un problème se pose également avec l'application de la loi de Darcy. En effet, les conditions de l'essai œdométrique classique dans lequel on mesure les caractéristiques de consolidation de l'argile correspondent à des gradients de pression extrêmement élevés. On peut alors se demander si les paramètres ainsi mesurés, et en particulier la perméabilité, peuvent être appliqués directement aux conditions du terrain où les gradients sont 100 à 1000 fois plus faibles. Par ailleurs, certains travaux ont conduit à suggérer l'existence d'un gradient limite au-dessous duquel la loi de Darcy ne serait plus applicable (Hansbo, 1960). Ce phénomène, qui pourrait avoir une importance capitale en fin de consolidation, n'est pas encore clairement défini mais il mérite une étude détaillée.

L'hypothèse 7 d'une relation linéaire entre contraintes et déformations volumétriques intervient à deux niveaux dans le développement et l'utilisation de la solution de Terzaghi; d'une part elle permet de passer des changements de volume aux variations de pressions interstitielles, pour transformer l'équation (6) et arriver à l'équation (11) d'autre part c'est grâce à elle qu'on peut assimiler l'expression (17) du degré de consolidation à la loi de variation du tassement total en fonction du temps. Or cette hypothèse n'est en général pas vérifiée dans la nature. On notera d'ailleurs que Terzaghi lui-même fait l'hypothèse contradictoire d'une relation linéaire entre les déformations volumétriques et le logarithme de la contrainte dans son calcul de l'amplitude des tassements (Terzaghi et Peck, 1965). On verra plus loin que cette hypothèse conduit dans certains cas à des erreurs importantes sur la loi de variation du tassement en fonction du temps.

Finalement, l'hypothèse 8 d'absence de toute viscosité structurale de l'argile est certainement contraire à la réalité. Les méthodes analytiques proposées pour tenir compte de cette viscosité (Koppejan, 1948 ; Gibson et Lo, 1961) ne sont pas entièrement satisfaisantes. L'utilisation de méthodes numériques a cependant conduit à une simulation plus convenable de la superposition des consolidations primaire et secondaire (Garlanger, 1972: Brucy, 1978).

II apparait donc clairement que les différentes hypothèses nécessaires au développement de la solution de Terzaghi ne sont pas totalement conformes au comportement physique réel d'une couche argileuse en consolidation. Par conséquent cette solution ne devrait être qu'approximative.

\section{But et principe de l'analyse par CONMULT}

Ayant mis en évidence certains aspects discutables des hypothèses utilisées dans la solution de Terzaghi, il est nécessaire de préciser dans quelle mesure ces hypothèses affectent la qualité des prévisions de consolidation. II faut pour cela avoir recours, en parallèle à la solution de Terzaghi, à une méthode numérique permettant d'éliminer les hypothèses les moins représentatives. Une telle méthode a été mise au point au LCPC, Paris, sous la forme du programme informatique CONMULT. Ce programme, décrit en détail par Magnan et al (1979), présente les caractéristiques essentielles suivantes:

- il permet la solution par la méthode des différences finies de l'équation unidimensionnelle:

$$
\frac{\partial u}{\partial t}=\frac{k(1+e) \sigma^{\circ}}{0.434 \gamma_{w} C_{c}} \quad \frac{\partial^{2} u}{\partial z^{2}}
$$

dans un système multicouche pouvant comporter jusqu'à 10 couches de caractéristiques différentes.

- Si l'hypothèse 1 de déformation unidimensionnelle reste utilisée, il est possible de se libérer de sa conséquence, soit $\Delta u=\Delta \sigma_{y}$, en imposant une distribution initiale des surpressions interstitielles indépendante des charges appliquées.

- Le programme, dans sa version la plus récente, comporte la prise en compte de la non-saturation et de la compressibilité possible du fluide interstitiel, éliminant aussi les hypothèses 2 et 3 .

- Une des qualités essentielles de CONMULT est d'être libéré des hypothèses 4 et 5 d'homogénéité et de constance des caractéristiques du sol. En effet la possibilité d'étudier un système multicouche élimine l'hypothèse d'homogénéité. Par ailleurs et surtout CONMULT permet de tenir compte des variations en cours de consolidation.

- de l'indice des vides:

- de la compressibilité, caractérisée par exemple par l'indice $\mathrm{C}_{\mathrm{C}}$ :

- de la perméabilité du milieu, suivant une loi du type :

$$
\mathrm{e}=\mathrm{A}+\mathrm{B} \lg \mathrm{k}
$$

De plus, par l'utilisation d'un système de sous-couches, on peut tenir compte des variations de ces propriétés en cours de consolidation à l'intérieur d'une couche initialement homogène. CONMULT dispense donc de l'utilisation du coefficient de consolidation $\mathrm{c}_{\mathrm{v}}$, dont on suppose dans la théorie de Terzaghi qu'il est une caractéristique constante de l'argile.

- CONMULT fait appel à la loi de Darcy, en écoulement unidimensionnel, mais en tenant compte des variations de $k$ et de longueur de drainage en cours de consolidation.

- Les tassements étant calculés pour chaque couche et sous-couche en fonction des variations de contrainte effective locale suivant la relation classique

$\Delta e=-C_{C} \Delta \lg \sigma^{\circ}$, CONMULT n'est pas affecté par l'hypothèse 7 de relation linéaire $e-\sigma^{\prime}$. En effet, le degré de consolidation ne sert qu'à contrôler le déroulement du calcul, mais pas à évaluer le tassement global du système étudié.

- Enfin, Brucy (1978) a mis au point une méthode de prise en compte de la consolidation secondaire, sur la base du modèle de Taylor-Bjerrum.

On voit donc que CONMULT ne contient finalement qu'une seule hypothèse contraignante, à savoir celle d'une consolidation unidimensionnelle. Le programme se prête donc très bien à une analyse détaillée de l'essai de consolidation œdométrique, et à la vérification de la validité de la théorie de Terzaghi dans ce cas.

\section{Chargement œdométrique d'une argile à $\mathrm{C}_{\mathrm{c}}=\mathrm{C}^{\text {ste }}$}

Ce cas de chargement simple, correspondant en principe à la théorie de Terzaghi, est celui que l'on rencontre lors des étapes de chargement dans l'essai œdométrique classique. On doit cependant distinguer les cas de l'argile surconsolidée peu compressible caractérisée par l'indice de gonflement $\mathrm{C}_{\mathrm{s}}$ et de l'argile normalement consolidée qui a au contraire un indice de compression $\mathrm{C}_{\mathrm{C}}$ élevé.

On sait que, selon Terzaghi, le coefficient de consolidation $c_{v}$ est constant durant un tel chargement et prend les valeurs correspondant à $\mathrm{C}_{\mathrm{S}}$ ou $\mathrm{C}_{\mathrm{C}}$ dans :

$$
C_{v}=\frac{k \sigma^{\prime}(1+e)}{0,434 C_{c} \gamma_{w}}
$$

En réalité $k$ et e varient au cours de la consolidation suivant les lois

$$
\begin{aligned}
& e=A+B \lg k \\
& e=e_{o}-C_{c} \lg \sigma / \sigma_{o}
\end{aligned}
$$

On peut donc, pour tenir compte de ces variations de $k$ et e, mettre l'équation (19) sous la forme: 


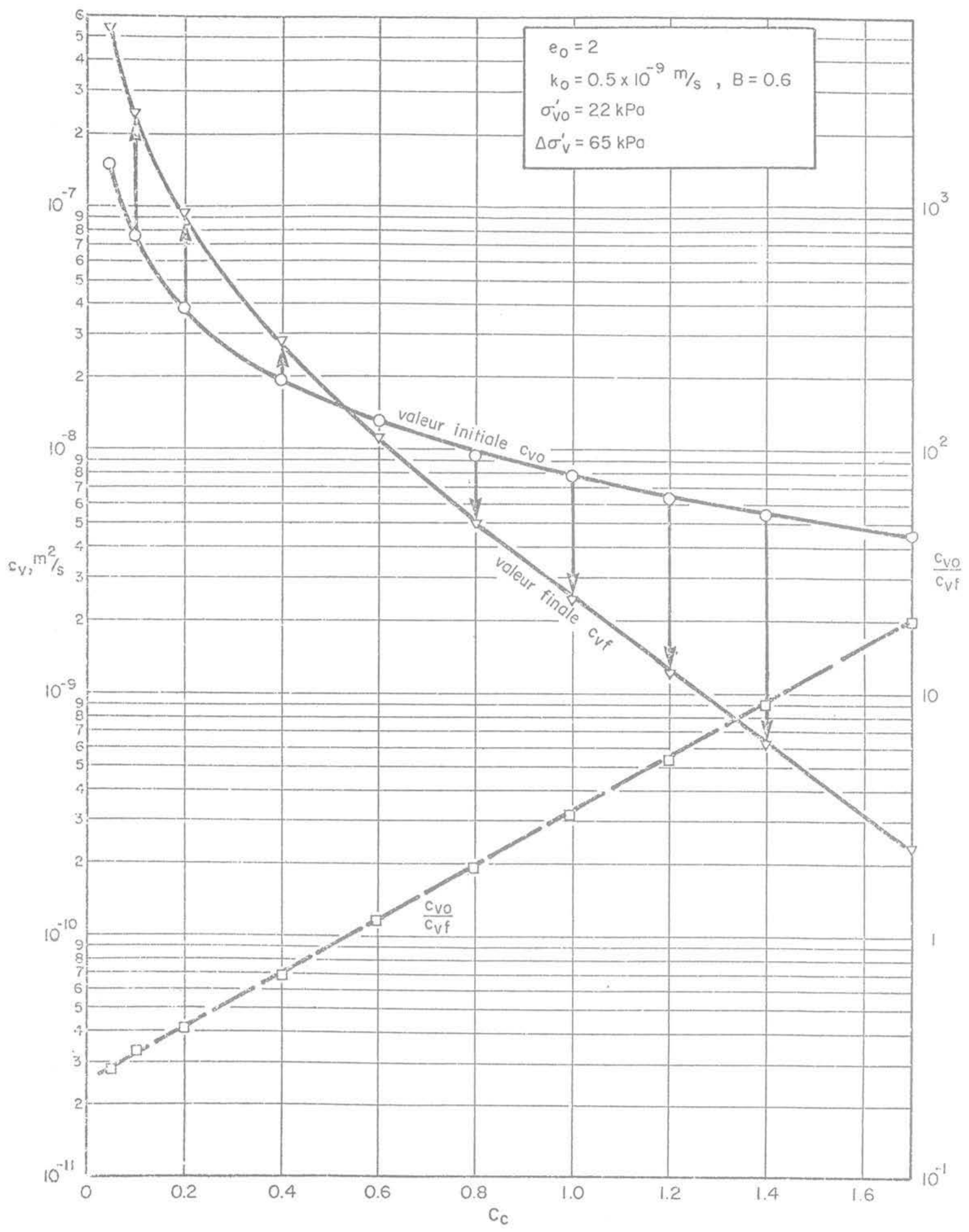

Fig. 1 Variations du coefficient de consolidation en cours de consolidation 


$$
\lg c_{v}=e\left(\frac{1}{B}-\frac{1}{C_{C}}\right)+\lg (1+e)-c^{\text {ste }}
$$

qui donne, en dérivant par rapport à e, la variation de $c_{v}$ en cours de consolidation, soit

$$
\frac{\Delta \lg c_{y}}{\Delta e}=\frac{1}{B}-\frac{1}{C_{C}}+\frac{1}{1+e}
$$

où e représente l'indice des vides final. On voit donc que, selon les valeurs relatives de $B$ et $C_{C}$, le coefficient $c_{V}$ pourra augmenter ou diminuer en cours de consolidation.

La figure 1 présente les variations en fonction de $C_{C}$ des valeurs initiales et finales de $c_{y}$ pour le cas d'un accroissement de charge $\Delta \sigma_{y}^{\prime}=65 \mathrm{kPa}$ appliqué à une argile normalement consolidée caractérisée par $e_{0}=2, k_{0}=0,5 \times$ $10^{-9} \mathrm{~m} / \mathrm{s}, \sigma_{v 0}^{\prime}=22 \mathrm{kPa}$ et $\mathrm{B}=0,6$. On note que $c_{y}$ augmente lorsque $C_{C}$ est inférieur à 0,5 et qu'il diminue considérablement pour les valeurs de $C_{C}$ supérieures à 0,5 , la loi de variation de ig $\left(c_{y o} / c_{y t}\right)$ étant une fonction linéaire de $\mathrm{C}_{\mathrm{C}}$. Les caractéristiques considérées sont celles d'une argile molle sensible pour laquelle l'indice de compressibilité peut prendre des valeurs typiques $C_{S}=0,05$ dans le domaine surconsolidé et $\mathrm{C}_{\mathrm{C}}=1,7$ dans le domaine normalement consolidé. Dans ces conditions, durant un chargement dans le domaine surconsolidé, $\mathrm{c}_{\mathrm{y}}$ augmentera par un facteur de l'ordre de 3 , ce qui se traduira par une accélération importante de la consolidation : c'est probablement la raison pour laquelle la mesure de $\mathrm{c}_{v}$ par la méthode classique fondée sur la théorie de Terzaghi est impossible dans une argile surconsolidée. Par contre, $c_{y}$ subira une réduction très importante lors d'un chargement dans le domaine normalement consolidé. On voit donc que, dans les deux cas, la théorie de Terzaghi ne sera pas correctement applicable.

Pour bien mettre en évidence ces phénomènes on a simulé à l'aide du programme CONMULT le comportement d'un échantillon de $1,95 \mathrm{~cm}$ de hauteur, drainé sur une seule face et possédant les caractéristiques citées plus haut, qui sont typiques de I'argile de Saint-Alban (Tavenas et Leroueil, 1977). Cet échantillon a été soumis à un accroissement de contrainte effective $\Delta \sigma_{v}^{\prime}=65 \mathrm{kPa}$ en supposant que l'argile était normalement consolidée et avait un indice de compression $\mathrm{C}_{\mathrm{C}}=1,7$. La figure 2 présente les variations du degré de tassement $U_{S}$, défini à partir du tassement de l'échantillon calculé par CONMULT, ainsi que les résultats obtenus par la solution de Terzaghi à partir des valeurs initiales et finales de e, $\mathrm{k}$ et $\sigma^{\prime}$.
On voit que le tassement réel se développe à une vitesse intermédiaire entre celles prévues par Terzaghi et correspondant à une valeur moyenne de $c_{y}$ de lordre de $0,5 \times$ $10^{-9} \mathrm{~m}^{2} / \mathrm{s}$ pour les degrés de consolidation supérieurs à 50 pour cent. La figure 3 présente les variations de $c_{v}$ en cours de consolidation aux quatre niveaux indiqués. On voit que la couche superficielle (point A) subit une diminution de $c_{y}$ très rapide au début de la consolidation pour jouer donc un rôle retardateur sur la consolidation des couches inférieures : l'examen combiné des figures 2 et 3 montre d'ailleurs que c'est le coefficient de consolidation $c_{v}$ de cette couche supérieure qui contrôle le coefficient de consolidation moyen de tout l'échantillon et la vitesse de tassement global. La figure 3 met par ailleurs clairement en évidence la non-vérification de l'hypothèse de base de Terzaghi concernant l'homogénéité du sol puisque à un même instant les valeurs de $c_{y}$ peuvent varier dans un rapport de 1 a 5 . Une simulation par CONMULT du chargement d'une argile surconsolidée $\left(C_{\mathrm{s}}=0,05\right)$ a confirmé l'augmentation de $c_{y}$ en cours de consolidation dans un rapport de 1 à 3 .

On voit donc que, même dans le cas simple d'un chargement par paliers d'une argile caractérisée par une valeur


phénomène réel, tant pour les argiles surconsolidées caractérisées par des valeurs faibles de $\mathrm{C}_{\mathrm{S}}$ que pour les argiles normalement consolidées où les valeurs de $C_{C}$ sont généralement supérieures à 1 . Les valeurs de $\mathrm{c}_{y}$ mesurées dans ce cas ne pourront être qu'approximatives puisqu'elles intègrent en fait les variations du coefficient de consolidation réel, dans l'espace et dans le temps.

\section{Chargement cedométrique d'une argile sur- consolidée}

II est très rare qu'une argile naturelle soit strictement normalement consolidée: les effets combinés du vieillissement, de la thixotropie, des variations de la nappe phréatique résultent tous en effet en une préconsolidation de I'argile, Lorsqu'une argile naturelle est soumise à un chargement, son comportement va donc être d'abord celui d'un matériau surconsolidé, puis, en cours de consolidation, celui d'un matériau normalement consolidé. On aura donc en cours de consolidation une variation très importante de l'indice de compression $C_{c}$ et, par voie de conséquence, du coefficient de consolidation $\mathrm{c}_{\mathrm{v}}$. Ce cas de chargement, qui correspond à tous les cas de chargement réel in situ, diffère donc sensiblement du chargement par palier

Fig. 2 Variations du degré de tassement $U_{S}$ en fonction du temps dans le cas où $C_{C}=1,7$





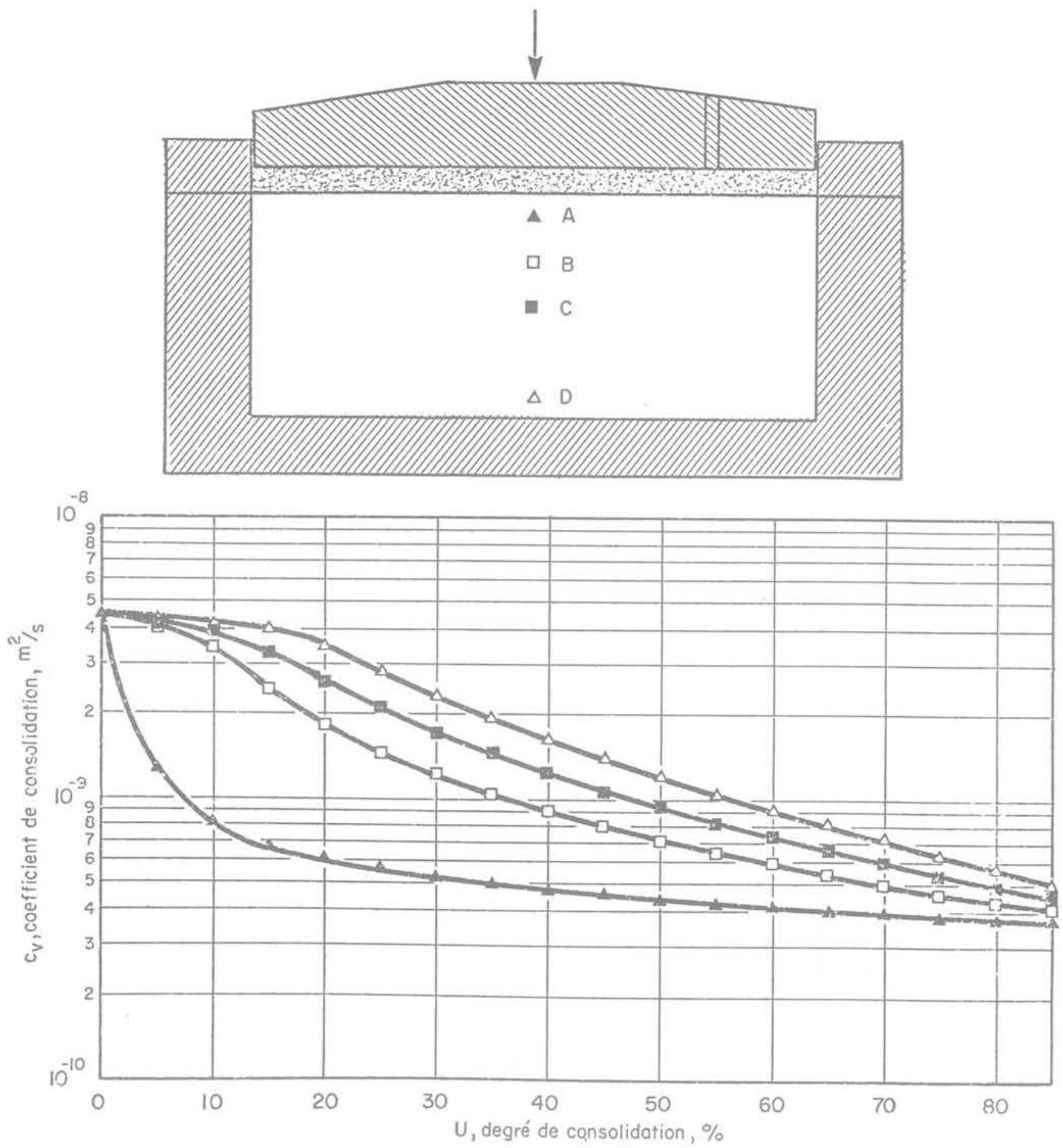

Fig. 3 Variations du coefficient de consolidation à différents niveaux dans l'échantillon en fonction du degré de consolidation global dans le cas où $C_{c}=1,7$

réalisé à l'œdomètre et mérite une étude particulière. Raymond (1966) a déjà considéré ce problème mais en ne tenant compte que de la variation de $\mathrm{C}_{\mathrm{c}}$ en cours de consolidation. Au contraire le programme CONMULT utilisé dans la présente étude permet une simulation complète des phénomènes réels en tenant compte non seulement des variations de $\mathrm{C}_{\mathrm{c}}$ mais aussi de la réduction de perméabilité en cours de consolidation.

Pour étudier ce problème on a décidé de simuler le chargement à l'œdomètre de l'argile de Saint-Alban à $3 \mathrm{~m}$ de profondeur sous les remblais d'essais (Tavenas et al. 1974). Cette argile est caractérisée par une contrainte effective verticale en place de $22 \mathrm{kPa}$ et un indice des vides initial de 2,0 , une pression de préconsolidation de 50
$\mathrm{kPa}$ et des indices $\mathrm{C}_{\mathrm{s}}=0,07$ et $\mathrm{C}_{\mathrm{c}}=1,7$. Sa perméabilité verticale est de l'ordre de $0,5 \times 10^{-9} \mathrm{~m} / \mathrm{s}$ à l'état naturel et des essais en laboratoire ont montré que cette perméabilité diminuait avec l'indice des vides suivant la loi $\Delta \mathrm{e}=0,6 \Delta \mathrm{Ig} \mathrm{k}$. La charge appliquée par le remblai correspond à un accroissement de contrainte effective verticale de $65 \mathrm{kPa}$ en fin de consolidation. La figure 4 présente la loi de compressibilité retenue dans cet intervalle de contraintes.

La figure 5 présente les variations du degré de consolidation en fonction du temps pour un échantillon œdométrique de $1,95 \mathrm{~cm}$ de hauteur, drainé à sa face supérieure suelement. Compte tenu de la loi effort-déformation suivie (fig. 4) l'hypothèse 7 de Terzaghi n'est plus vérifiée et l'équation (17) donnant la vitesse de dissipation des pres- 




Fig. 4 Relation $e-\lg \sigma_{v}^{\prime}$ prise en compte pour l'étude de la consolidation dans le cas où $\sigma_{v o}^{\prime}<\sigma_{p}^{\prime}<\sigma_{v o}^{\prime}+\Delta p^{\prime}$

Fig. 5 Variations des degrés de consolidation $U$ et de tassement $U_{s}$ en fonction du temps dans le cas où $\sigma_{\text {vo }}^{\prime}<\sigma_{p}^{\prime}<\sigma_{\text {vo }}^{\prime}+\Delta p$

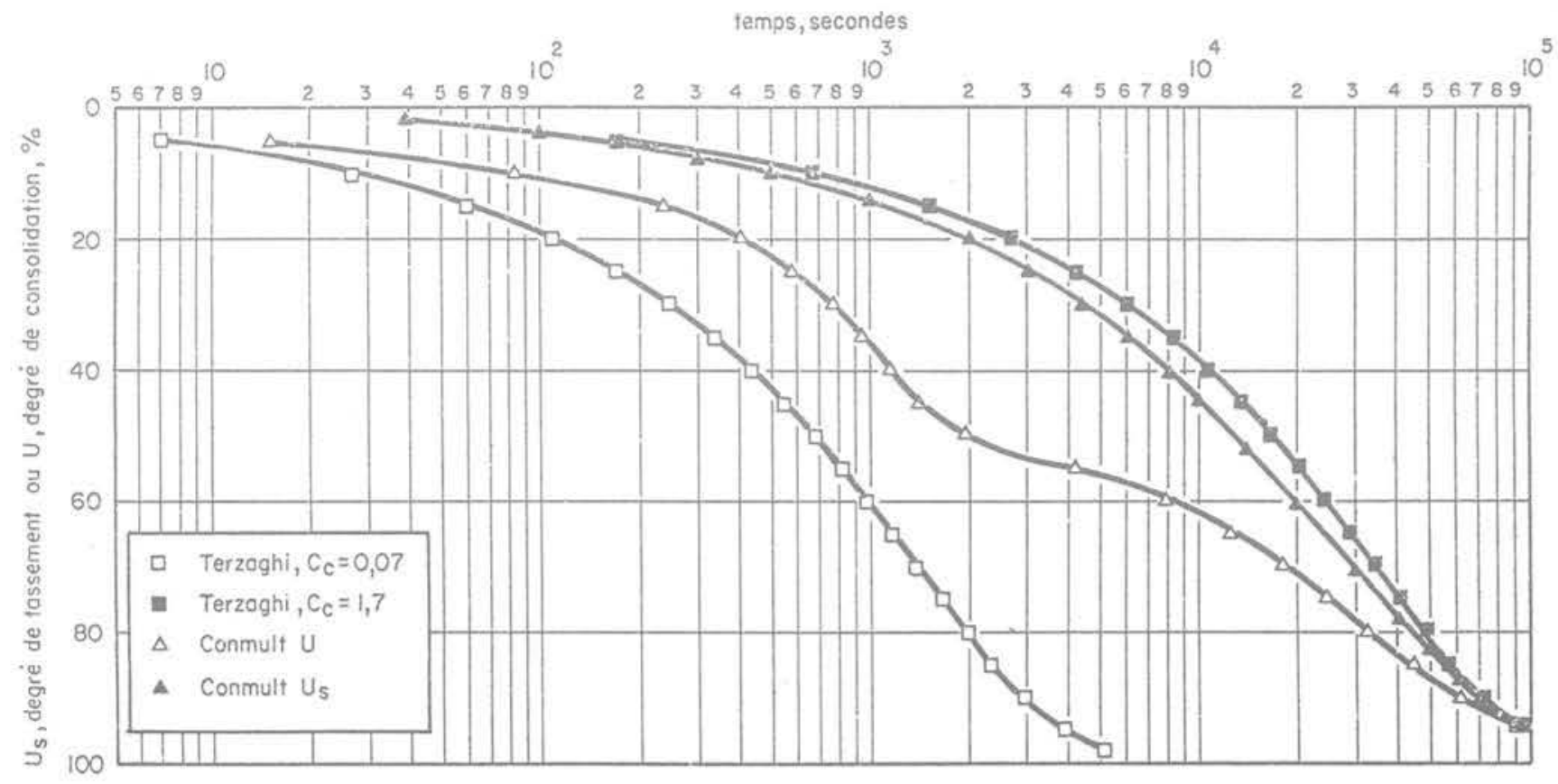




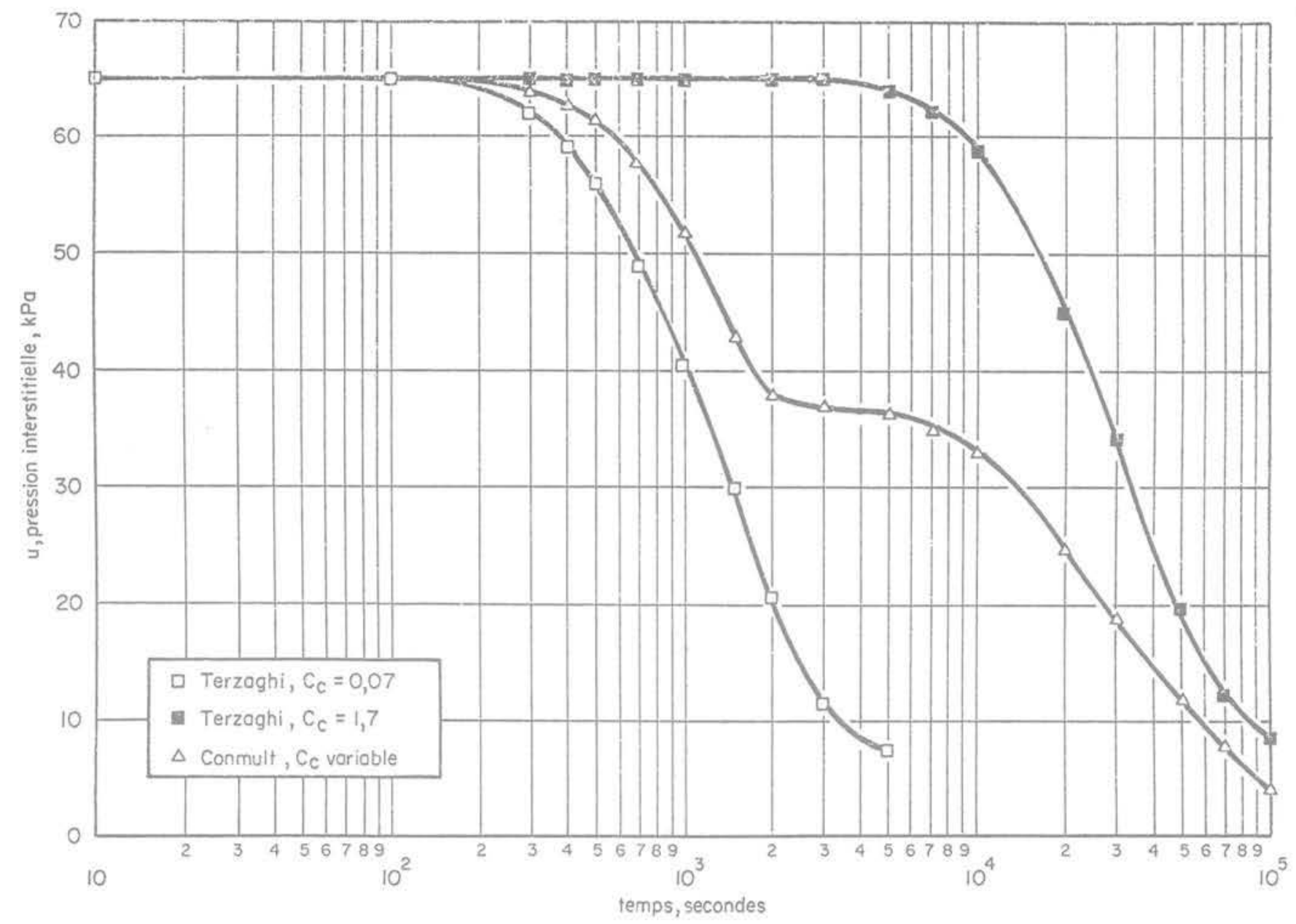

Fig. 6 Variations de la surpression interstitielle à la base non drainée de l'échantillon

Fig. 7 Variations de la contrainte effective verticale à la base non drainée de l'échantillon

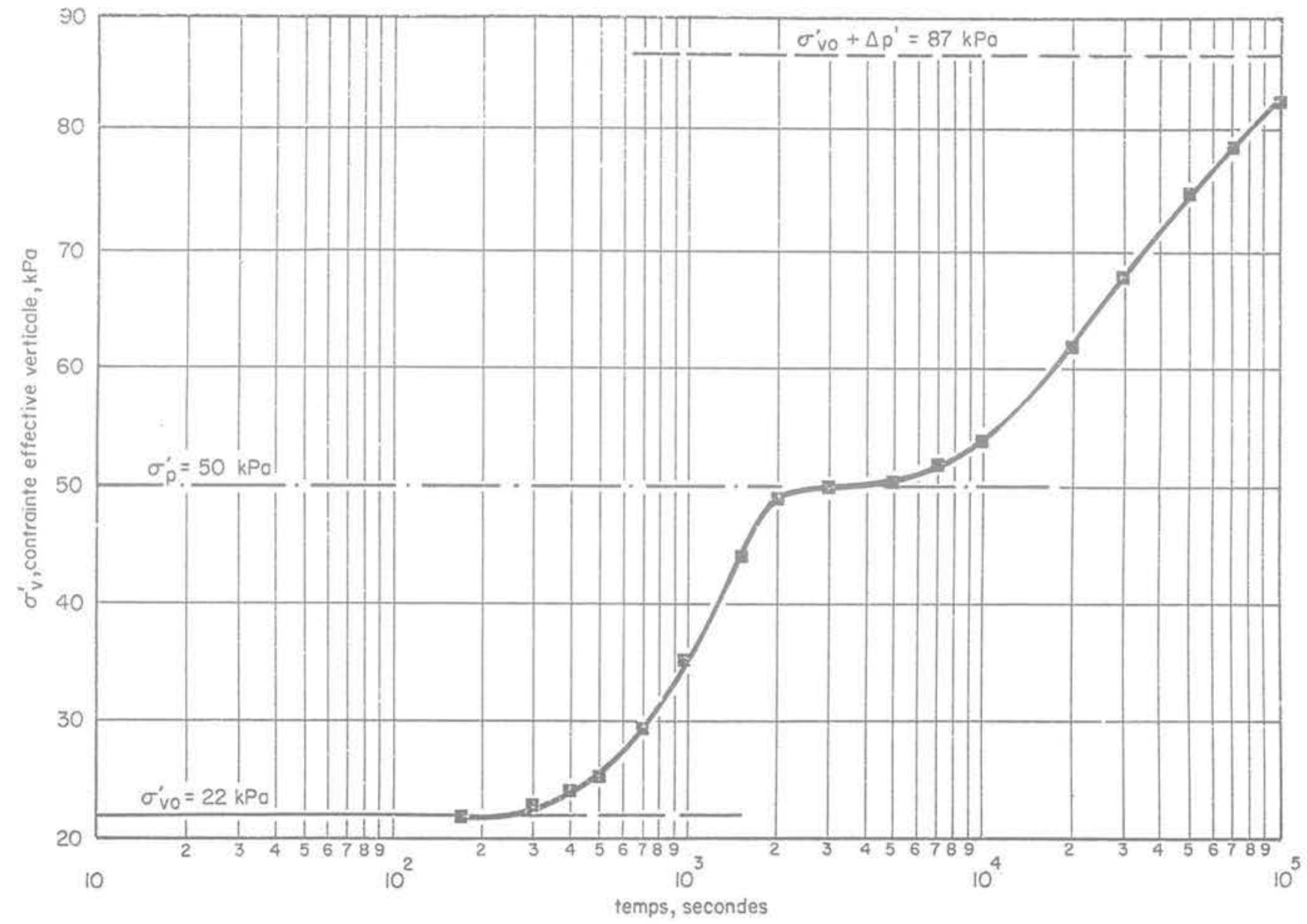


disiribution des contraintes effeciives

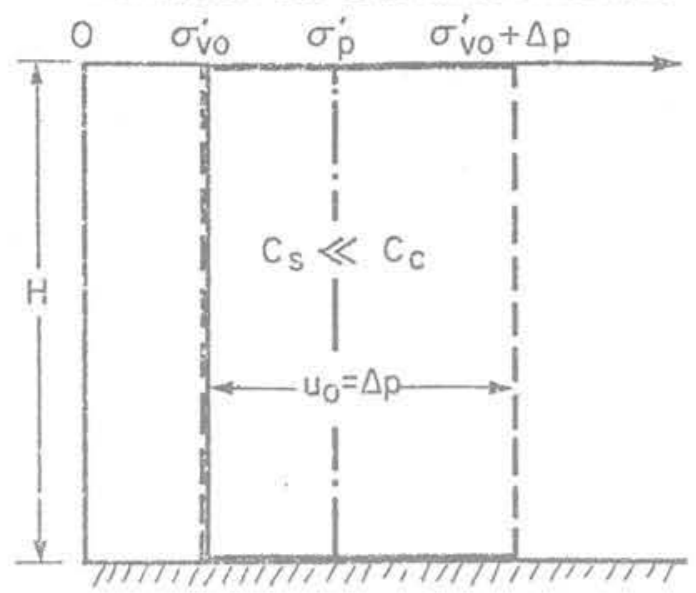

modèle de comportement

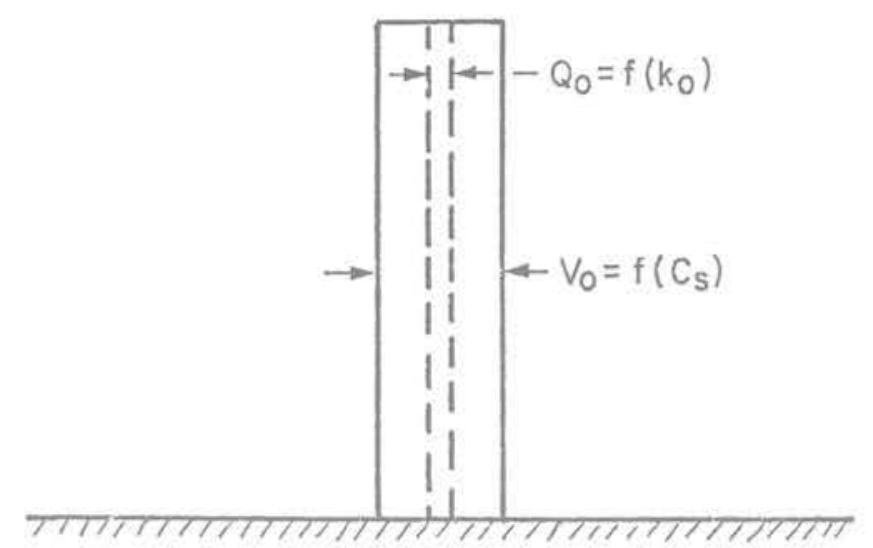

a) instant $t=0$


b) instant $t_{1}$
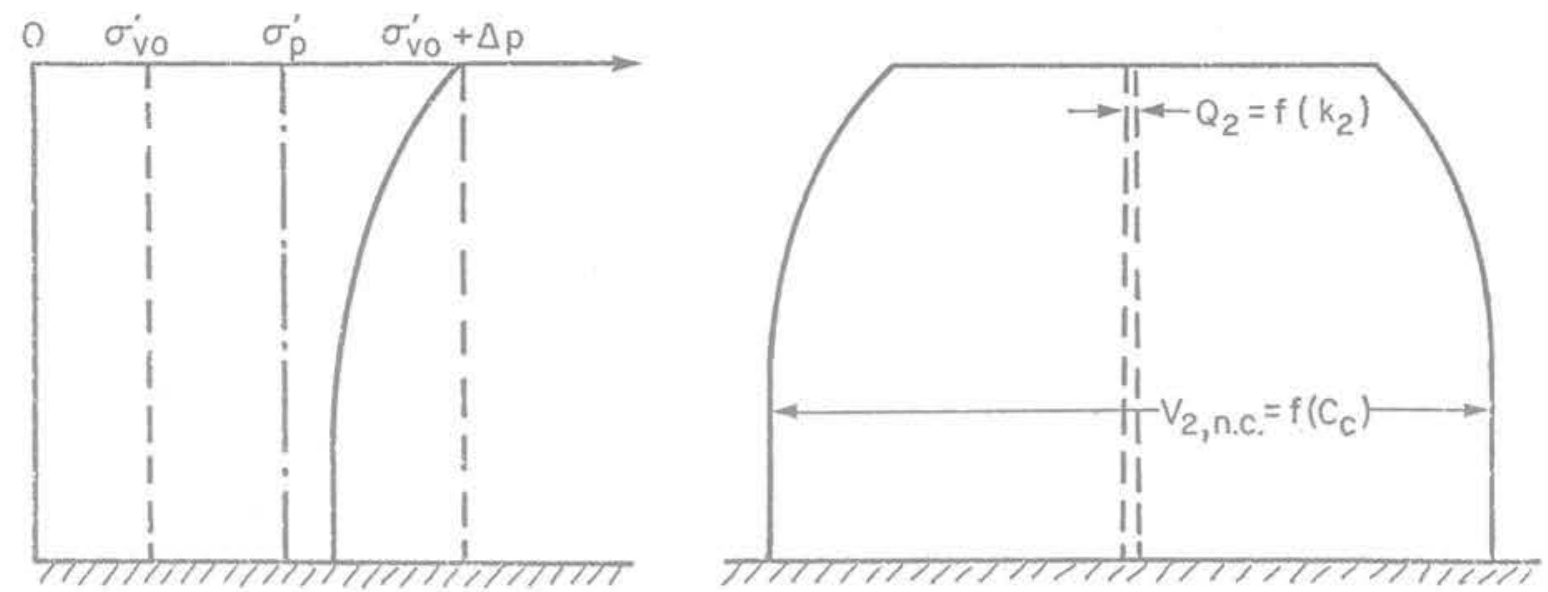

c) instant $t_{2}$

Fig. 8 Principe du comportement en cours de consolidation d'une argile initialement surconsolidée soumise à $\sigma_{v o}^{\prime}+\Delta p>\sigma_{p}^{\prime}$ 
sions interstitielles ne permet plus de prévoir l'accroissement des tassements en fonction du temps. Effectivement le degré de consolidation U déterminé à partir des pressions interstitielles calculées par CONMULT à différents niveaux dans l'échantillon suivant une équation de la forme de (16), diffère sensiblement du degré de tassement $U_{s}=\Delta h / \Delta h_{\text {final }}$. On a également reporté sur la figure 5 les solutions de Terzaghi pour $C_{\mathrm{s}}=0,07$ et pour $\mathrm{C}_{\mathrm{C}}=1,7$ et sur la base des valeurs initiales de e, $\sigma^{\prime}$ et $k$. On voit que la variation de $U_{S}$ en fonction du temps obtenue par CONMULT prend une forme assez semblable à la solution de Terzaghi avec $C_{C}=1,7$, la différence maximum étant de l'ordre de 6 pour cent de degré de consolidation. Cette similitude, déjà notée par Raymond (1966) doit être considérée avec prudence comme on le notera plus loin. Au contraire la variation du degré de consolidation $U$ en fonction du temps présente une forme tout à fait différente des solutions de Terzaghi : initialement elle s'éloigne progressivement de la solution avec $C_{\mathrm{s}}=0,07$, présente une discontinuité entre 50 et 60 pourcent de consolidation, pour finalement se rapprocher de la solution avec $C_{C}=1,7$. Pour mieux comprendre cette forme particulière de $U=f(t)$ on peut étudier la dissipation des pressions interstitielles à la base non drainée de l'échantillon. Les variations de $u$ en fonction du temps calculées selon les deux solutions de Terzaghi et par CONMULT sont présentées à la figure 6 . On retrouve qualitativement le mêrne comportement que pour le degré de consolidation global avec un éloignement initial progressif de la solution avec $\mathrm{C}_{\mathrm{S}}=0,07$, un palier qui dure de 2000 à 5000 secondes et pendant lequel la pression interstitielle se maintient à peu près constante à $37 \mathrm{kPa}$, puis finalement un rapprochement progressif vers la solution avec $C_{c}=1,7$. Le blocage temporaire de la pression interstitielle peut s'expliquer par le passage de l'argile de l'état surconsolidé à l'état normalement consolidé. En effet, d'après les variations de contrainte effective verticale à la base de l'échantillon (fig. 7). on voit que le palier de la courbe de consolidation se produit lorsque la contrainte effective verticale devient égale à la pression de préconsolidation de l'argile $\sigma_{p}^{\prime}=50 \mathrm{kPa}$. A ce moment, au niveau considéré, la compressibilité de l'argile augmente considérablement (ici elie est multipliée par $C_{C} / C_{s}=24$ ) ce qui se traduit par une augmentation brusque du volume d'eau à évacuer et, donc, par un ralentissement de la dissipation de la pression interstitielle. Ce phénomène, logique au niveau élémentaire, ne suffit pas à lui seul pour expliquer le comportement global de l'échantillon tel que présenté à la.figure 5 ; en effet il devrait se produire à des instants différents aux différents niveaux de l'échantillon et se traduire par un déplacement continu de $U$ de la solution avec $C_{S}=0,07$ vers la solution avec $\mathrm{C}_{\mathrm{C}}=1,7$.

La figure 8 présente schématiquement le comportement de l'échantillon en cours de consolidation. A l'application de la charge, à l'instant $t=0$, on a $\Delta u=\Delta p$, et l'argile est caractérisée par ses propriétés initiales $e_{0}, \sigma_{\mathrm{vo}}, \mathrm{k}_{\mathrm{o}}$ et $\mathrm{C}_{\mathrm{S}}$ : l'échantillon est en condition surconsolidée homogène, la consolidation se faisant à une vitesse contrôlée par le volume à évacuer $V_{0}=f \cdot\left(C_{s}\right)$ et le débit possible $Q_{0}=f\left(k_{0}\right)$. A mesure que la consolidation se développe les pressions interstitielles se dissipent à partir de la face drainée et la contrainte effective augmente. A l'instant $t_{1}$ la contrainte effective est supérieure à $\sigma_{p}$ entre la face drainée et la profondeur $\mathrm{z}$. Dans cette zone l'argile a pris des caractéristiques normalement consolidées ; le volume d'eau à évacuer y est accru dans le rapport $C_{c} / C_{S}$ alors que le débit possible a diminué par suite de la réduction de $k$ associée à la diminution de l'indice des vides. Le débit disponible sert alors en priorité à évacuer l'eau de cette zone normalement consolidée située près de la surface drainante et il va y avoir un blocage de la consolidation de la zone surconsolidée inférieure. Avec le développement de la consolidation l'épaisseur $z_{A}$ de la zone normalement consolidée va croître, pour finalement être égale à l'épaisseur de l'échantillon. A partir de ce moment tout l'échantilIon sera à l'état normalement consolidé mais il ne sera pas pour autant homogène puisque ses caractéristiques $\sigma^{\prime}$, e, $k$ et donc $c_{v}$ seront variables d'un point à l'autre.
Le développement d'une zone "bouchon 》 normalement consolidée à proximité de la surface drainante modifie la forme des isochrones, tel qu'indiqué à la figure 9. Pour un même degré de consolidation global, on a, par rapport aux isochrones déterminés selon la théorie de Terzaghi, une diminution plus lente des pressions interstitielles près de la surface drainante dans la couche normalement consolidée oủ $c_{v}$ est petit. Par contre, dans la zone centrale surconsolidée où le processus de consolidation est temporairement bloqué on note un rééquilibrage des pressions interstitielles à l'intérieur de cette zone; on atteint même une condition de gradient nul entre la base et la mi-hauteur de l'échantillon pour $U=50 \%$. En considérant la variation de la forme et de la position des isochrones ou des courbes de distribution de $\sigma_{y}$ en fonction de la profondeur (fig. 10) on peut comprendre le processus de formation du palier de la courbe $U=f(t)$. On voit que, pour un degré de consolidation de 45 pour cent, le tiers seulement de la couche est déjà en état normalement consolidé, alors que pour un degré de consolidation de 55 pour cent $\sigma_{\mathrm{y}}^{\prime}$ est

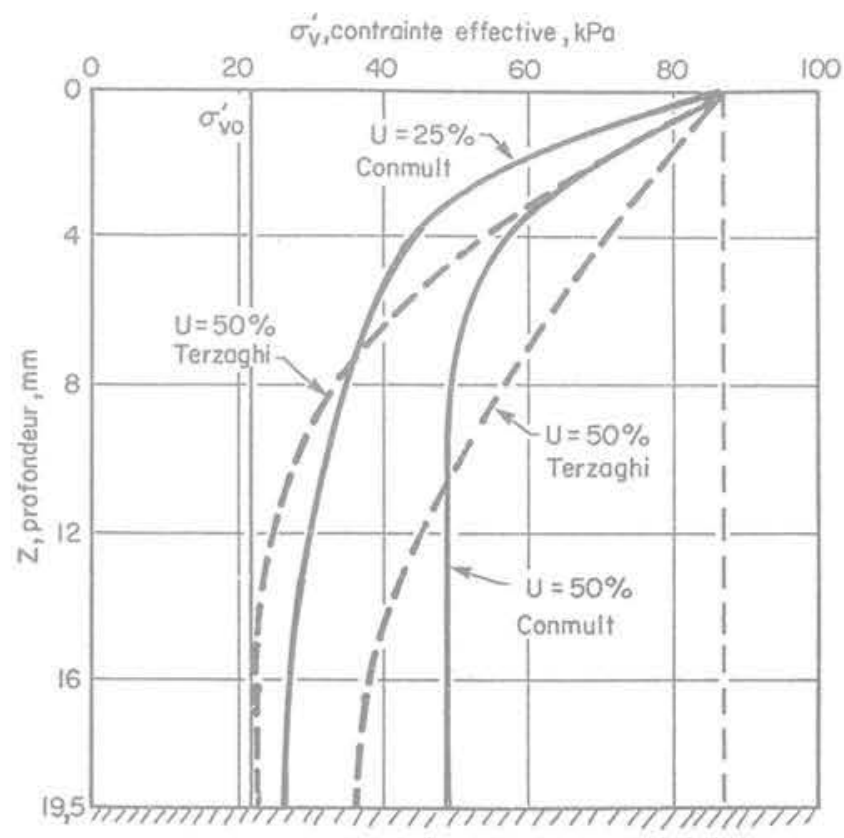

Fig. 9 Déformation des isochrones dans une argile initialement surconsolidée soumise à $\sigma_{v o}^{\prime}+\Delta p>\sigma_{p}^{\prime}$

Fig. 10 Isochrones au voisinage du passage de l'ensemble de l'échantillon à l'état normalement consolidé






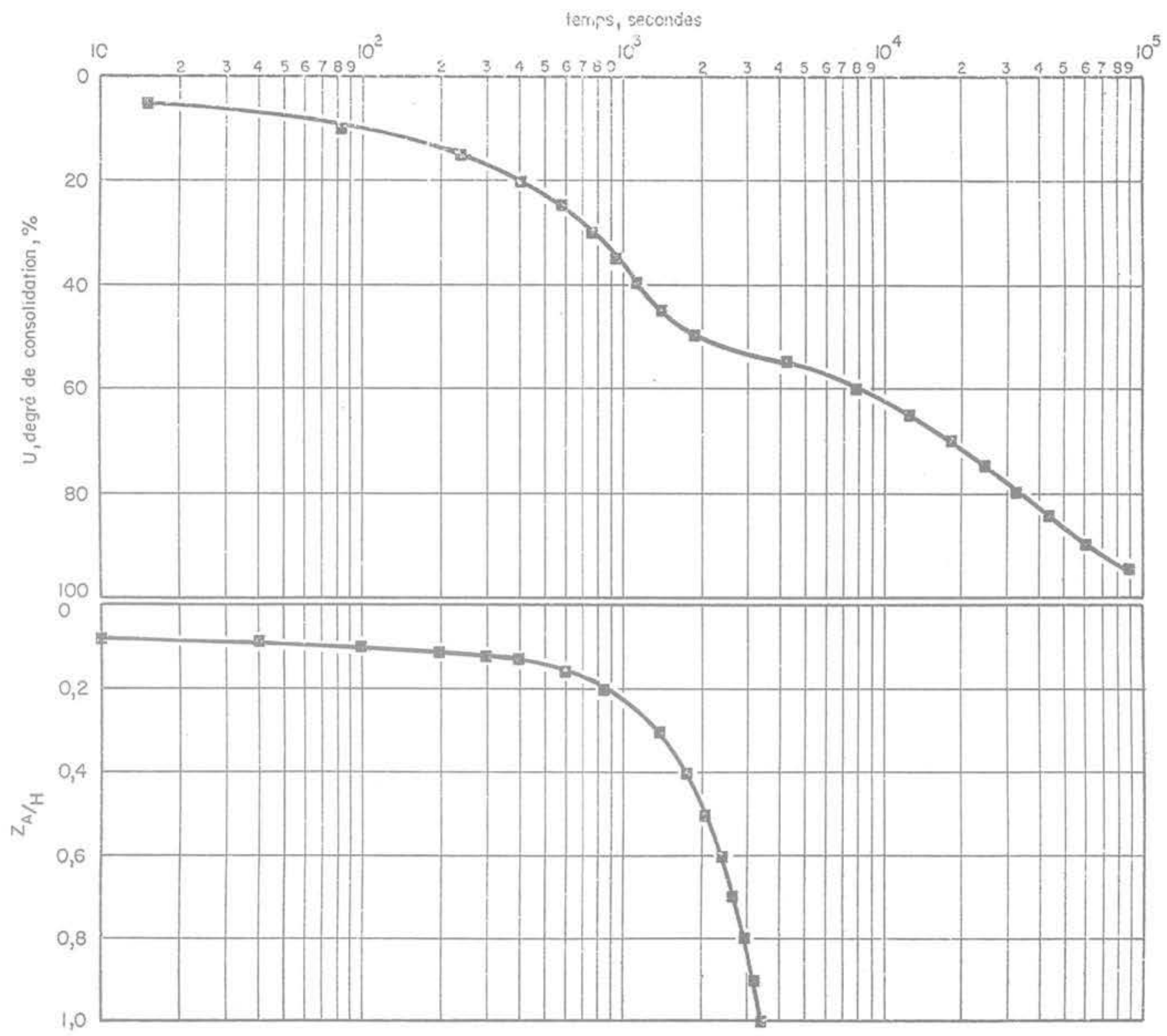

Fig. 11 Variations en fonction du temps du degré de consolidation $U$ et de la hauteur d'échantillon qui se trouve à l'état normalement consolidé

supérieure à $\sigma_{\mathrm{p}}^{\prime}$ dans l'ensemble de l'échantillon. Cela se traduit par une augmentation rapide du volume d'eau à évacuer et par un ralentissement important de la consolidation. La figure 11 met en évidence ce phénomène en présentant les variations en fonction du temps de la hauteur relative d'échantillon en état normalement consolidé et du degré de consolidation $U$; on note que le palier de $U$ $=f(t)$ se produit pendant i'augmentation rapide de ${ }^{2} A$.

La durée du palier doit être fonction du surcroît d'eau à évacuer lorsque l'ensemble de l'échantillon devient normalement consolidé, c'est-à-dire du rapport $\mathrm{C}_{\mathrm{c}} / \mathrm{C}_{\mathrm{s}}$. Pour vérifier cette hypothèse on a fait deux analyses par CONMULT en gardant tous les paramètres identiques au cas précédent mais en prenant $C_{c}=0,85$ dans un cas et $C_{C}=3,4$ dans l'autre. La figure 12 présente les variations de la pression interstitielle à la base de l'échantilion en fonction du temps pour les trois cas étudiés. La dissipation initiale de $\mu$ est d'autant plus rapide que $C_{0}$ est petit : l'etfet de « bouchon » produit par le passage à l'état normalement consolidé de la couche supérieure est en effet fonction du rapport $\mathrm{C}_{\mathrm{C}} / \mathrm{C}_{\mathrm{s}}$. Par ailleurs la longueur du palier de la courbe $u=f(t)$ varie en fonction de $C_{C}$. Pour préciser cette variation on a défini la durée du palier comme étant le temps nécessaire à la diminution de la pression interstitielle de 38 à $37 \mathrm{kPa}$. La figure 13 présente les variations de $\Delta t$ en fonction de $C_{c} / C_{S}$. On note que $\Delta t$ augmente à peu près linéairement avec $\mathrm{C}_{\mathrm{C}} / \mathrm{C}_{\mathrm{s}}$ jusqu'à une valeur de lordre de 20 au-delà de laquelle la croissance de $\Delta t$ devient exponentielle. On peut remarquer par ailleurs que si on rapporte la durée $\Delta t$ du palier au temps nécessaire pour atteindre 95 pour cent de dissipation de $u$, le palier représente une proportion du temps de consolidation décroissant de 2,8 pour cent pour $\mathrm{C}_{\mathrm{C}} / \mathrm{C}_{\mathrm{s}}=12,1$ à 1 pour cent pour $\mathrm{C}_{\mathrm{c}} / \mathrm{C}_{\mathrm{s}}=48,6$. 


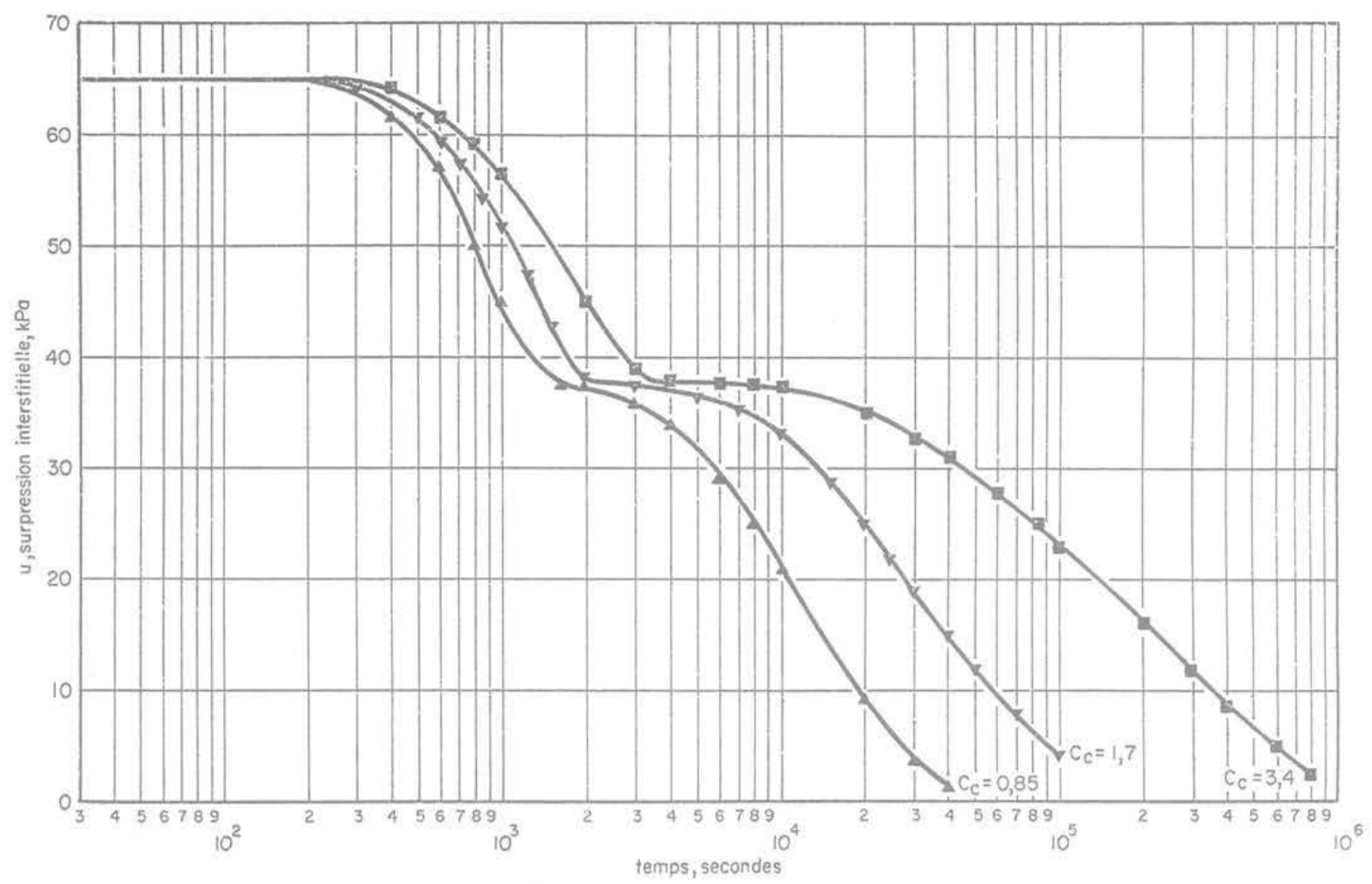

Fig. 12 Influence du rapport $C_{c} / C_{s}$ sur la dissipation de la surpression interstitielle à la base non drainée de l'échantillon

Fig. 13 Variation de la durée du palier de surpression interstitielle à la base de la courbe en fonction de $C_{c} / C_{s}$



Fig. 14 Variation du degré de tassement $U_{s}$ en fonction du degré de consolidation $U$






\section{Conclusion}

Pour arriver à une formulation simple et à une solution du problème du développement en fonction du temps du tassement d'une couche d'argile, Terzaghi (1925) a été amené à faire une série d'hypothèses très restrictives, dont la plupart sont peu représentatives de la réalité physique, Cependant, la simplicité de la méthode a conduit à son utilisation quasi universelle et, avec l'usage, les limitations résultant des hypothèses ont été perdues de vue.

La mise au point du programme d'analyse numérique CONMULT, qui libère de la nécessité de faire toute hypothèse autre que celle d'une consolidation unidimensionnelle et qui représente en particulier les variations réelles des propriétés de compressibilité et de perméabilité de l'argile en cours de consolidation, a permis une analyse détaillée de la qualité de la solution de Terzaghi.

Dans le cas d'un chargement par palier, l'accroissement de $c_{y}$ en cours de consolidation dans l'argile surconsolidée, et sa réduction très importante dans l'argile normalement consolidée, font que la théorie de Terzaghi donne des résultats peu représentatifs. Inversement, les valeurs de $c_{v}$ déduites d'essais oedométriques interprétés à partir de cette théorie représentent des valeurs moyennes correspondant à des conditions de contrainte, perméabilité et indice des vides peu claires.

En conclusion, dans le cas classique de chargement d'une argile initialement surconsolidée soumise à une contrainte effective finale supérieure à sa pression de préconsolidation, le comportement en cours de consolidation diffère très nettement des prévisions obtenues par la théorie de Terzaghi. Le passage rapide à l'état normalement consolidé des zones situées à proximité des surfaces drainantes conduit à la formation d'un "bouchon » qui ralentit globalement la consolidation et qui modifie considérablement la forme des isochrones. Par ailleurs, cette modification de la forme des isochrones, associée au changement de comportement de l'argile lorsqu'elle devient normalement consolidée, conduit à la formation d'un palier dans la courbe de variation du degré de consolidation $U$ en fonction du logarithme du temps. Enfin le degré de consolidation U déterminé à partir des pressions interstitielles n'est plus représentatif de la progression du tassement, tel que montré à la figure 14. On est donc forcé de conclure que la théorie de Terzaghi n'est pas adaptée à l'étude de ce cas de chargement. Pourtant on a noté (fig. 5) que la progression du tassement en fonction du temps prenait une forme assez semblable à celle déduite de la théorie de Terzaghi en prenant pour le calcul de $c_{y}$ les valeurs initiales de $\sigma$, e et $\mathrm{k}$ et l'indice de compression ${ }_{c}$ de l'argile normalement consolidée. II faut bien voir que ceci n'implique pas que le $c_{y}$ de l'argile normalement consolidée contrôle la vitesse de tassement. En effet, on a montré (fig. 1 et 2) que le coefficient de consolidation variait énormément en cours de consolidation dans une argile normalement consolidée, entraînant un développement du tassement beaucoup plus lent que celui prédit à partir de la valeur initiale de $c_{y}$. Le coefficient de consolidation apparent qui correspond à la vitesse de tassement dans le cas présent n'a aucune réalité physique et n'est finalement que le résultat de l'intégration des coefficients de consolidation plus grands dans la zone surconsolidée qui contrôlent le tassement initial et des $c_{v}$ beaucoup plus petits dans l'argile normalement consolidée qui gouvernent la phase finale de consolidation.

Dans le cas plus général d'un chargement amienant une argile initialement surconsolidée dans un état final normalement consolidé, le comportement réel diffère totalement de celui prédit par la théorie de Terzaghi. Le passage des zones proches des surfaces drainantes à l'état normalement consolidé dès le début de la consolidation produit la formation d'un "bouchon " qui ralentit la consolidation et modifie la forme des isochrones. De ce fait, il ne saurait être question d'utiliser la théorie de Terzaghi pour analyser la dissipation de pressions interstitielles ou le développement du tassement de couches élémentaires dans une fondation argileuse soumise à un tel chargement. Par ail- leurs le développement du degré de consolidation $U$ présente une discontinuité lorsque l'ensemble de la couche argileuse passe en état normalement consolidé et on ne peut pas déduire la progression des tassements de ce degré de consolidation.

Dans ces conditions il convient d'abord de réaliser que le coefficient de consolidation $c_{y}$ n'est pas une propriété réelle de l'argile, mesurable, constante et utilisable comme le veut la pratique courante actuelle. $c_{v}$ n'est que la combinaison des paramètres $\sigma^{\circ}, e, k$ et $C_{C}$ qui sont tous variables en cours de consolidation. Si on veut donc correctement définir le comportement hydrodynamique d'une argile, ce $n^{\prime}$ est pas $c_{Y}$ qu'il faut mesurer, mais bien les lois de variations de $e_{,} \mathrm{k}$ et $\mathrm{C}_{c}$ en fonction de la contrainte effective $\sigma$ '. D'autre part, la théorie de Terzaghi fait appel à trop d'hypothèses contraires à la réalité physique des phénomènes pour conduire à des prévisions fiables de la consolidation des fondations argileuses. II apparait au contraire nécessaire d'avoir recours à des méthodes d'analyse numérique, telles que le programme CONMULT, qui libèrent de l'obligation de formuler de telles hypothèses, et qui permettent une simulation complète du comportement réel de l'argile.

\section{Remerciements}

La présente étude a été réalisée dans le cadre du projet de coopération franco-québécoise liant le Laboratoire Central des Ponts et Chaussées de Paris et le Groupe de Géotechnique de l'Université Laval à Québec. Le financement en a été assuré par une bourse France-Québec, par un octroi FCAC du Ministère de l'Education du Québec et par l'octroi numéro A.7724 du Conseil National de Recherches du Canada.

\section{Annexe}

L'équation rigoureuse de la consolidation unidimensionnelle en variables d'Euler s'écrit généralement sous la forme (Schlosser, 1973):

$$
(1+e)^{2} \frac{\partial}{\partial z}\left[\frac{k}{(1+e) \gamma_{w}} \quad \frac{d \sigma^{\prime}}{d e} \quad \frac{\partial e}{\partial z}\right]=-\frac{\partial e}{\partial t}
$$

avec les notations

$\mathrm{e}=$ indice des vides à l'instant $\mathrm{t}$,

$z=$ cote du point considéré à l'instant $t$

$k=$ coefficient de perméabilité à l'instant $t$ et à la cote $\mathrm{z}$

$\sigma^{\prime}=$ contrainte effective verticale à l'instant t et à la cote $z$,

$\gamma_{\mathrm{W}}=$ poids volumique de l'eau,

$\mathrm{t}=$ temps.

En variables de Lagrange, la même équation s'écrit :

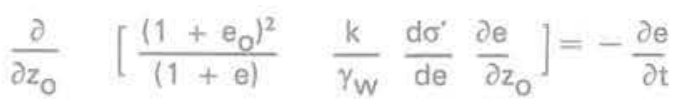

avec les notations

$z_{0}=$ cote initiale du point considéré

$e_{0}=$ valeur initiale de l'indice des vides à la cote $z_{0}$.

Ces deux équations sont non linéaires puisqu'elles dépendent entre autres de l'indice des vides actuel e. Pour rendre l'équation (A.2) linéaire et semblable à l'équation de Terzaghi, il est nécessaire de supposer $k$ constant, $\frac{d \sigma}{d e}$ constant et e constant dans l'expression $(1+e)$. On peut noter de plus que le simple fait de supposer le produit $\frac{\left(1+e_{o}\right)^{2}}{1+e} \frac{k}{\gamma_{w}} \frac{d \sigma^{\prime}}{d e}$ constant dans l'équation (A.2) rend linéaire l'équation différentielle qui donne le tassement mais pas celle qui permet de calculer la surpression interstitielle. 


\section{Références bibliographiques}

BRUCY M. (1978) - Analyse de la consolidation primaire et secondaire des argiles Champlain par Commult. Thèse de M.Sc., département de Génie civil, Université Laval, Québec, Canada.

FREDLUND D.L. (1976) - Density and compressibility characteristics of air-water mixtures. Canadian Geotechnical Journal, Vol. 13 (4) : 386-396.

GARLANGER J.E. (1972) - The consolidation of soils exhibiting creep under constant effective stress. Geotechnique, Vol. 22 (1): 71-78

GIBSON R.E., LO, K.Y. (1961) - A theory of consolidation for soils exhibiting secondary compression. Norwegian Geotechnical Institute, Publication number 41

HANSBO S. (1960) - Consolidation of clay, with special reference to influence of vertical sand drains. Swedish Geotechnical Institute, Proc. 18.

KOPPEJAN, A.W, (1948) - A formula combining the Terzaghi load-compression relation ship and the Buisman secular time effect. Proc. 2nd ICSMFE, Rotterdam, Vol, 3 ; 3237.

LEROUEIL S., TAVENAS F., TRAK B., LA ROCHELLE P., ROY M. (1978) - Construction pore pressures in clay foundations under embankments - Part I - the Saint-Alban test fills. Canadian Geotechnical Journal, Vol. 15 (1) : 54-65.
MAGNAN, J.-P., DANG, M.-T. (1977). - Theoretical and experimental analysis of the compressibility of pore fluid in a nearly saturated clayey soil. Proc. Int. Symp. on Geot. Aspects of soft clays, AIT, Bangkok, : 675-690.

MAGNAN J.-P., BAGHERY S., BRUCY M., TAVENAS F. (1979), - Etude numérique de la consolidation unidimensionnelle avec prise en compte des variations de la perméabilité et de la compressibilité du sol, du fluage et de la non saturation. (à paraitre dans le bulletin de liaison des LPCParis).

RAYMOND G.P. (1966). - Consolidation of slightly overconsolidated soils. ASCE Journ. Soil Mech. and Found. Div., Vol. 92 (SM5) : 1-19.

SCHLOSSER F. (1973) - Hypothèses ot théories pour la prévision des tassements des remblais sur sols compressibles. Buil. de Liaison des Laboratoires des Ponts et Chaussées, $n^{\circ}$ spécial $T$ : 26-57,

SKEMPTON A.W., BJERRUM L. (1957), - A contribution to the settlement analysis of foundation on clay. Geotechnique, Vol, 7 (2) : 168-178.

TAVENAS F., CHAPEAU C., LA ROCHELLE P., ROY M. (1974). - Immediate Settlements of three test embankments on Champlain clay. Canadian Geotechnical Journal, Vol. 11 (1) : 109-141.

TAVENAS F., LEROUEILS. (1977), - Effects of stresses and time on yielding of clays. Proc. 9th ICSMFE, Tokyo, Vol. 1 : $319-326$

TERZAGHI K. (1925). - Erdbaumechanik. Deuticke Ed., Vienna.

TERZAGHI K., PECK R.B. (1965), - Mécanique des sols appliquée. Dunod, Paris. 
